DOI https://doi.org/10.30525/978-9934-26-075-9-19

\title{
SEPSIS IN OBSTETRIC AND GYNECOLOGICAL PRAXIS SPECIFICS, FEATURES AND NEW WAYS TO SOLVE THE PROBLEM
}

\author{
Ostapiuk L. R. \\ Candidate of Medical Sciences, \\ Obstetrician-Gynecologist \\ Lviv Regional Public Health Center \\ Lviv, Ukraine
}

The issue of preserving women's reproductive health is the key factor in ensuring their health, the birth of healthy children and the health of the nation. But in the process of pre-pregnancy training, pregnancy, childbirth and the postpartum period there are many provoking factors that can cause a negative impact at each of these stages. Consequently, it is necessary to create a clear strategy for successful pregnancy and the birth of a healthy newborn baby. $99 \%$ of maternal mortality occurs in low-income countries. The main causes of death are postpartum hemorrhage, preeclampsia and obstetric sepsis [1].

Nevertheless, obstetric sepsis is one of the leading causes of maternal mortality not only in low-income countries. At first glance, this fact is quite unpredictable. The state of the constant improvement of diagnostic and treatment tactics as well as the further increase of the frequency of postpartum purulent-inflammatory complications is controversial. Although the reasons for low- and high-income countries are not the same, the fact remains: in the 21 st century, the problem of postpartum purulent-inflammatory diseases remains extremely relevant and needs an urgent and successful solution. In high-income countries, there is a significant increase in surgical activities within obstetrics that contributes to the increased incidence of postpartum purulent-inflammatory diseases and sepsis. This is facilitated by a social pattern of behaviour that delays a woman's planned pregnancy. As a rule, in high-income countries, women plan pregnancy after the successful building of their career in the late reproductive age. But at this time, most patients have extragenital pathologies, including severe ones. Also, relevant is the problem of infertility and the use of assisted reproductive technologies, induced multiple pregnancies that is an additional load on the body of the pregnant woman. Also in high-income countries, the problem of increasing the incidence of obesity and diabetes among pregnant women is significant. 
Patients with diabetes are at risk for postpartum purulent-septic complications, including postpartum endometritis (PPE).

For explaining the link between diabetes and the protracted course of purulent-inflammatory diseases effectively, it is necessary to appeal to the indepth analysis of the pathogenesis of their occurrence and development.

The aim of this study is to substantiate the pathogenetic concept of purulent-inflammatory diseases and sepsis, as well as to explain the specific features of their occurrence in patients with diabetes.

The study material consists of samples of dilutions of blood serum (BS) by bacterial culture, sugar broth, 20\% albumin solution and distilled water (in vitro study). These results are important for understanding the possible changes of the fluorescence spectra (FS) of BS in patients with various diseases and treatment tactics [2]. The clinical research centre of the study was the purulent-septic centre of the Ambulance Municipal City Clinical Hospital (Lviv) and the Department of Gynaecology №2 of Vinnytsia Council Clinical Hospital №2. The in vivo component included 15 patients with sepsis and 190 new mothers with PPE. The control group included 40 women with uncomplicated flow of the postpartum period.

Methods of investigation: clinical, laboratory (general and biochemical blood tests, general urine test, smear on the flora), instrumental (uterus and ovarian sonography, histologic analysis of metroaspirate), statistical methods (logistic regression and ROC-analysis). In addition to standard research methods, all patients underwent the examination of samples of BS by using the method of fluorescence spectroscopy (MFS). The Luminescence Laboratory of the Department of Experimental Physics of the Ivan Franko National University of Lviv was an experimental research centre. Women with single pregnancy, who had a histologically confirmed diagnosis of PPE in the postpartum period were involved in the research. These women were informed beforehand and gave an informed consent for participation in the aforementioned study. The postpartum period after multiple pregnancies, the period after antenatal death of the foetus, decompensated somatic illnesses, the presence of primary immunodeficiency among postpartum women, the presence of HIV infection, tuberculosis (pulmonary and extra-pulmonary), diabetes and a presence of oncological pathology were all considered to be exclusion criteria.

Results of the research. In patients with diseases, which are accompanied with endogenous intoxication, there are changes in the system of albumin molecules. They are based on the fact that they have the ability to complex. In these diseases, part of the albumin molecules in the blood of patients is blocked by toxins. As a result, there are two types of albumin molecules in 
their blood: normal (concentration $-\mathrm{X}$ ) and blocked by toxins (pathological; concentration - 1-X). Pathological albumin molecules lose the ability to perform their basic functions, esp. those of transportation and detoxification. The new definition of sepsis is based on the existence of $\mathrm{X}^{*}$, i.e. the borderline minimum concentration of albumin in patients with purulent-inflammatory diseases and sepsis. If $\mathrm{X}$ is greater than $\mathrm{X}^{*}$, it ensures the vital functions of the organism. If $\mathrm{X}$ less than $\mathrm{X}^{*}$, here comes exitus letalis.

Now we will dwell in detail on the results of the study of FS of BS of a patient with a severe PPE in the dynamics (Fig. 1, Table 1). In the same figure and in table. 1 for comparison, the corresponding results for a patient with sepsis who was treated in 2002 are depicted [3].

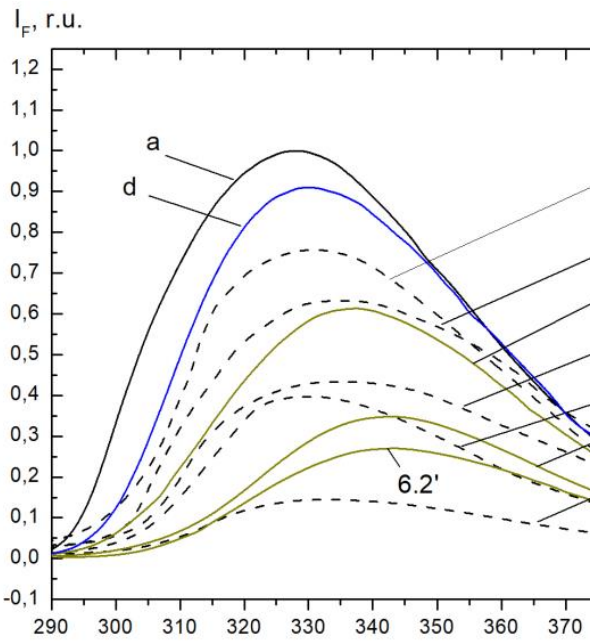

Fig. 1. Fluorescence spectra of serum of a woman after childbirth with endometritis in dynamics (6.1 - 14.02.; 6.2 - 17.02., 6.2'-17.02. ), a woman with uncomplicated course of postpartum period (2), patient with sepsis $\left(1^{\prime}, 2^{\prime}, 3^{\prime}, 4,^{\prime} 5^{\prime}\right)$ and $20 \%$ donor albumin (a).

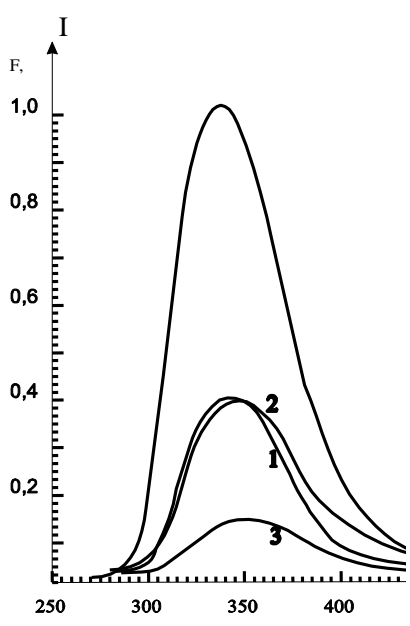

Fig. 2. FS of BS of patient 3 with sepsis and diabetes: 1 - 03.06; 2 - 05.06; 3 - 06.06 and donor BS. $\lambda_{\mathrm{ex}}=280 \mathrm{~nm}$. 
Table 1

\begin{tabular}{|c|c|c|c|c|c|c|c|c|c|c|}
\hline № & $\begin{array}{c}\text { Albumi } \\
\mathrm{n}\end{array}$ & 2 & 6.1 & 6.2 & $6.2^{\prime}$ & $1^{\prime}$ & $2^{\prime}$ & $3^{\prime}$ & $4^{\prime}$ & $5^{\prime}$ \\
\hline $\begin{array}{c}\text { Dat } \\
\mathrm{e}\end{array}$ & 14.02 & $\begin{array}{c}14.0 \\
2\end{array}$ & $\begin{array}{c}14.0 \\
2\end{array}$ & $\begin{array}{c}17.0 \\
2\end{array}$ & $\begin{array}{c}17.0 \\
2\end{array}$ & 3.06 & 5.06 & 6.06 & 7.06 & $\begin{array}{c}10.0 \\
6\end{array}$ \\
\hline$\lambda_{\max }$ & 330.1 & $\begin{array}{c}330 . \\
1\end{array}$ & $\begin{array}{c}343 . \\
1\end{array}$ & $\begin{array}{c}337 . \\
1\end{array}$ & $\begin{array}{c}343 . \\
1\end{array}$ & $\begin{array}{c}335 . \\
2\end{array}$ & $\begin{array}{c}335 . \\
2\end{array}$ & $\begin{array}{c}334, \\
1\end{array}$ & $\begin{array}{c}331, \\
6\end{array}$ & 331 \\
\hline $\mathrm{I}_{\mathrm{F}}$ & 1 & 0.91 & 0.35 & 0.61 & 0.27 & 0.63 & 0.43 & 0.16 & 0.40 & 0.76 \\
\hline
\end{tabular}

Table 2

\begin{tabular}{|c|c|c|c|c|c|c|c|c|c|c|}
\hline № & $\begin{array}{c}\text { Albumi } \\
\mathrm{n}\end{array}$ & 6.1 & 6.2 & 6.3 & 6.4 & 6.5 & $1^{\prime}$ & $3^{\prime}$ & $4^{\prime}$ & $5^{\prime}$ \\
\hline $\begin{array}{c}\text { Dat } \\
\mathrm{e}\end{array}$ & 14.02 & $\begin{array}{c}14.0 \\
2\end{array}$ & $\begin{array}{c}17.0 \\
2\end{array}$ & $\begin{array}{c}20.0 \\
2\end{array}$ & $\begin{array}{c}22.0 \\
2\end{array}$ & $\begin{array}{c}25.0 \\
2\end{array}$ & 3.06 & 6.06 & 7.06 & $\begin{array}{c}10.0 \\
6\end{array}$ \\
\hline$\lambda_{\max }$ & 330.1 & $\begin{array}{c}343 . \\
1\end{array}$ & $\begin{array}{c}348 . \\
2\end{array}$ & $\begin{array}{c}339 . \\
0\end{array}$ & 336 & 334 & $\begin{array}{c}335 . \\
2\end{array}$ & $\begin{array}{c}334, \\
1\end{array}$ & $\begin{array}{c}331, \\
6\end{array}$ & 331 \\
\hline $\mathrm{I}_{\mathrm{F}}$ & 1.0 & 0.35 & 0.24 & 0.38 & 0.55 & 0.68 & 0.63 & 0.16 & 0.40 & 0.76 \\
\hline
\end{tabular}

Somatic and gynecological anamneses were burdened in this woman, and she also had other risk factors for PPE. This patient showed a significant decrease in fluorescence intensity down to 0.35 r.u. and a noticeable longwavelength shift of the fluorescence band. After manual vacuum aspiration of the uterine wall, antibacterial and uterotonic therapy, the patient's condition improved. After correcting treatment in the next experiment, a marked increase in the $\mathrm{I}_{\mathrm{F}}$ of $\mathrm{BS}$ of this patient up to 0.6 r.u. was recorded, and the longwavelength shift of the fluorescence band was leveled. Without correcting treatment tactics, the patient's condition could deteriorate in principle (curve 6.2') with a subsequent transition to a septic state (curve $1^{\prime}$ ), as in the above-mentioned patient with sepsis.

Due to the proper level of treatment of patients with endometritis (even in severe form), the septic condition seems to be almost unattainable. For the correction of treatment tactics, infusions of $100-150 \mathrm{ml}$ of $20 \%$ albumin solution could be prescribed. In low-income countries, septic conditions are possible in principle in the case of inadequate treatment. But even in this case mortality could be avoided by adjusting the treatment accordingly and prescribing infusions of $20 \%$ albumin solution. If the patient had passed to the state of 6.2' in our case, prescribing up to $150-200 \mathrm{ml}$ of albumin several times could have ensured its recovery. It is very important to monitor the treatment process within the MFS and patients' health. Undoubtedly, the high 
qualification of doctors and control over the treatment process within the MFS were also very important in this case.

Fig. 2 presents FS of BS of a patient with sepsis and diabetes mellitus. Unfortunately, the scenario of this disease was not optimistic. In this case, in addition to pathological albumin molecules, the number of glycosylated albumin molecules was also increased. In healthy individuals, about $6 \%$ of albumin molecules in BS are glycosylated. And in patients with diabetes due to the presence of hyperglycemia there are $9-12 \%$ of glycosylated molecules. Thus, it is possible that patients with diabetes have a tendency to develop purulent-septic processes and their long course. This problem is currently very topical in high-income countries due to the growing number of obese and diabetic patients, including pregnant women, while pregnant women with diabetes are at risk for postpartum purulent-inflammatory diseases.

The condition of our patient deteriorated rapidly during the observation period, despite the surgery and intensive antibiotic therapy, which can be explained by the presence of a number of severe comorbidities and her old age, accompanied with a gradual decrease in the intensity of the fluorescence band (Fig.2 , curves 1, 2, 3). The patient died as a result of an advanced process of the generalization of the infection and multiple organ failure. This scenario could have been prevented if the patient had obtained additionally at least $200 \mathrm{ml}$ of albumin solution on June 3-5, followed by its daily use until possible recovery. Unfortunately, there is no guarantee that this scenario would be successful.

Conclusions. According to the proposed pathogenetic model of development of purulent-inflammatory diseases and sepsis in the complex treatment of such patients, it is advisable to diagnose and monitor their condition within the MFS and to use albumin infusion in their complex treatment.

\section{References:}

1. Ostapiuk L. Diagnostic and Therapeutic Model of Sepsis and PurulentInflammatory Diseases. International Journal of Clinical Medicine. 2019. № 10. P. 577-595. https://doi.org/10.4236/ijcm.2019.1011047.

2. Bulavenko O., et al. Modelling Changes in Blood Serum at Different Diseases and Therapeutic Measures. Biomedical and Biosocial Anthropology. 2013. № 20. P. 8-14.

3. Ostapiuk L., et al. Problems and Challenges to Women's Reproductive Health in the 21th Century. Acta Scientific Women's Health Special Issue. 2021. № 3. P. 70-87. 